\title{
Benign lymph node inclusions mimicking metastatic carcinoma
}

\author{
C J Fisher, S Hill, R R Millis
}

\begin{abstract}
Aims-To draw attention to nonneoplastic inclusions in axillary lymph nodes removed from women with primary breast cancer which may be mistaken for metastases.

Methods-Five examples of non-malignant inclusions were detected in axillary lymph nodes removed from women with mammary carcinoma. Immunohistochemical staining for CAM $5 \cdot 2$ and $\mathbf{S 1 0 0}$ markers, as well as morphological assessment were performed.

Results-Three of the five cases comprised naevus cells and two heterotopic epithelial elements. One of each was initially mistaken for metastatic carcinoma. Naevus cells in the capsule of axillary nodes are often arranged as lines of small, round cells and may readily be misinterpreted as metastatic lobular carcinoma. Heterotopic epithelial elements, in the form of tubules, can easily be mistaken for metastasis from an infiltrating ductal carcinoma.

Conclusions-Awareness of the occasional occurrence of non-neoplastic nodal inclusions will help avoid misdiagnosis. If immunohistochemical markers for epithelial cells are used to screen lymph nodes for metastasis, such lesions could be detected more frequently. It is important to combine immunohistochemistry with morphological assessment and the use of a panel of antibodies in histological diagnosis.
\end{abstract}

$(\Im$ Clin Pathol 1994;47:245-247)

Recently we have seen five cases of breast carcinoma in which non-malignant inclusions were found in the accompanying axillary lymph nodes. In three cases benign naevus cells were found and two consisted of heterotopic epithelial elements.

Although uncommon, such findings are well recognised. ${ }^{1-6}$ Indeed, four of the cases occurred within a space of five months, during which time 101 axillary clearances were examined. Several examples of benign lymph node inclusions have been reported before. As two of our cases were, initially, thought to represent metastatic carcinoma, we considered it worthwhile re-emphasising the phenomenon, especially as nowadays lymph nodes may be examined by immunohistochemistry using epithelial markers which will highlight some of these small heterotopic elements.

\section{Case reports}

CASE 1

A 39 year old woman had a lump removed from her left breast, together with a palpable axillary lymph node. Microscopy showed an infiltrating lobular carcinoma of classic type, and it was initially thought that the lymph node contained metastatic tumour cells within the capsule (fig 1). After further morphological and immunohistochemical examination, which showed negative CAM 5.2 and positive $S 100$ staining, the capsular elements were identified as benign naevus cells. It is important to use more than one marker because, as in this case, mammary carcinomas can often show $S 100$ positivity.

\section{CASE 2}

A 71 year old woman underwent an excision biopsy and axillary clearance after needle core diagnosis of mammary carcinoma of classic lobular type. One of the 13 dissected axillary nodes contained benign naevus cells in the capsule. No metastatic tumour was found. In this case the nature of the cells was immediately recognised.

\section{CASE 3}

A 70 year old woman had a right mastectomy for a $2 \mathrm{~cm}$ infiltrating carcinoma which had been diagnosed on biopsy and contained areas of both pleomorphic lobular and ductal carcinoma NOS (grade III). The 19 axillary nodes dissected were free of tumour but one node contained naevus cells not only within the capsule but also in the parenchyma and within a blood vessel in the surrounding fat (fig 2). Interestingly, a polypoid benign intradermal naevus was present near the nipple; this, too, showed vascular invasion.

\section{CASE 4}

A 55 year old woman had a suspicious lesion on mammography. The area was localised and an infiltrating grade I ductal carcinoma with focal tubule formation (tubular mixed) was removed. Epithelial lined tubules were found in one of 21 axillary nodes (fig 3). These were initially interpreted as metastatic carcinoma, but closer examination showed that they resembled benign epithelial elements in the breast and were morphologically and cytologically different from the infiltrating malignant 

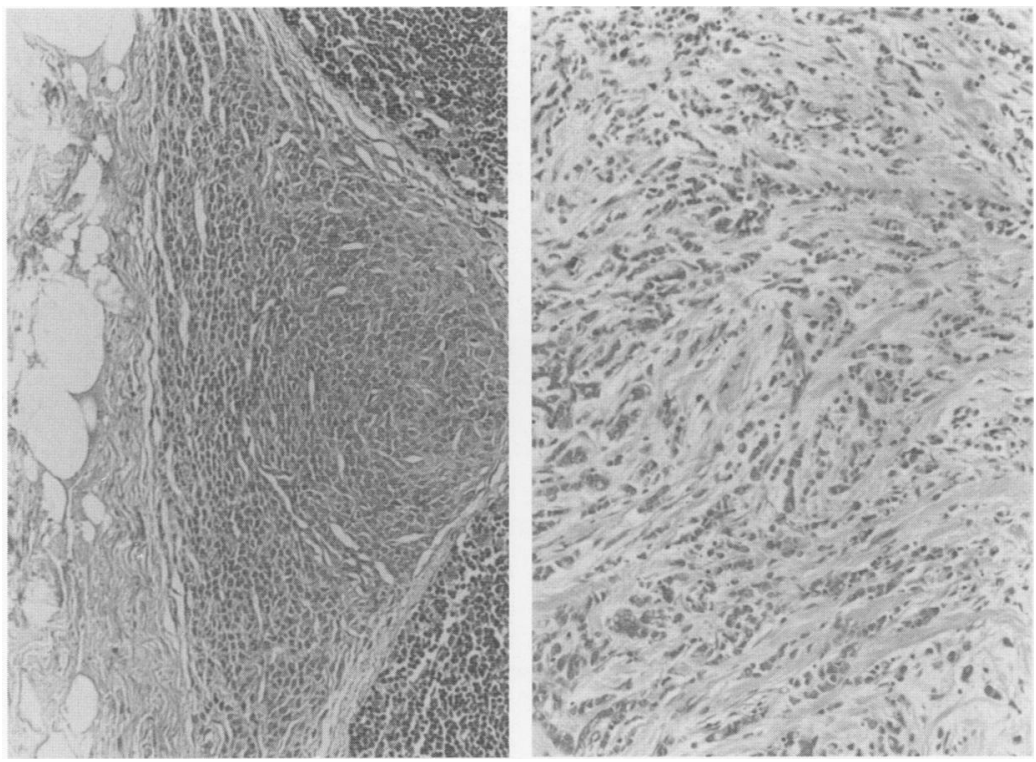

Figure 1 Case 1. (A) Benign naevus cells in capsule of lymph node; (B) infiltrating lobular carcinoma in ipsilateral breast.

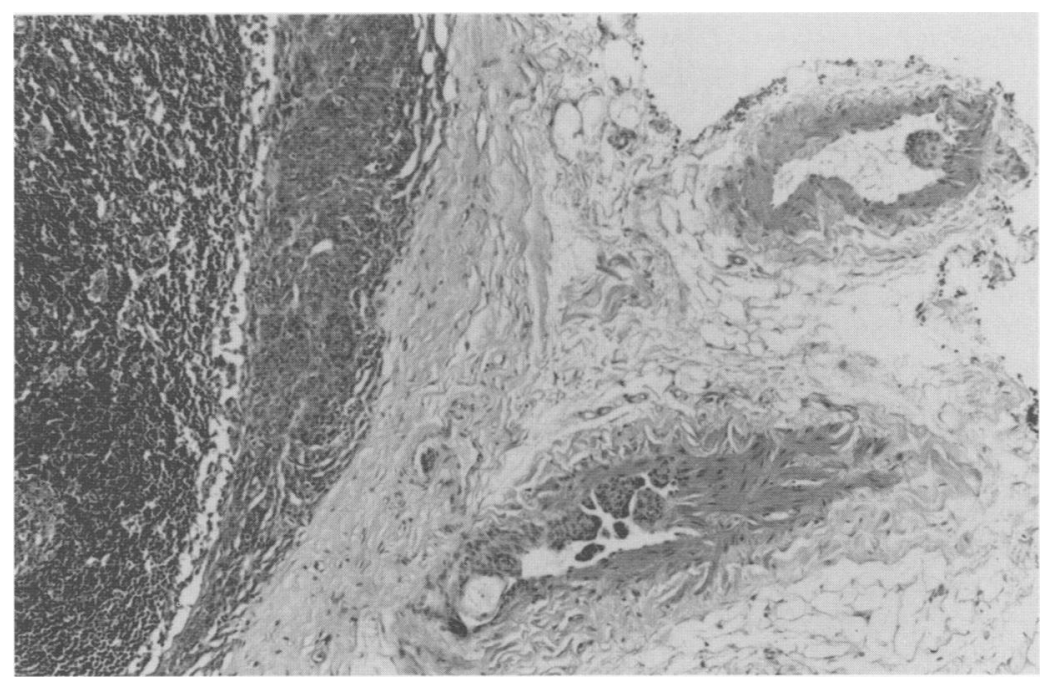

Figure 2 Case 2. Benign naevus cells within capsule of lymph node and forming polypoid protrusions within adjacent blood vessels.
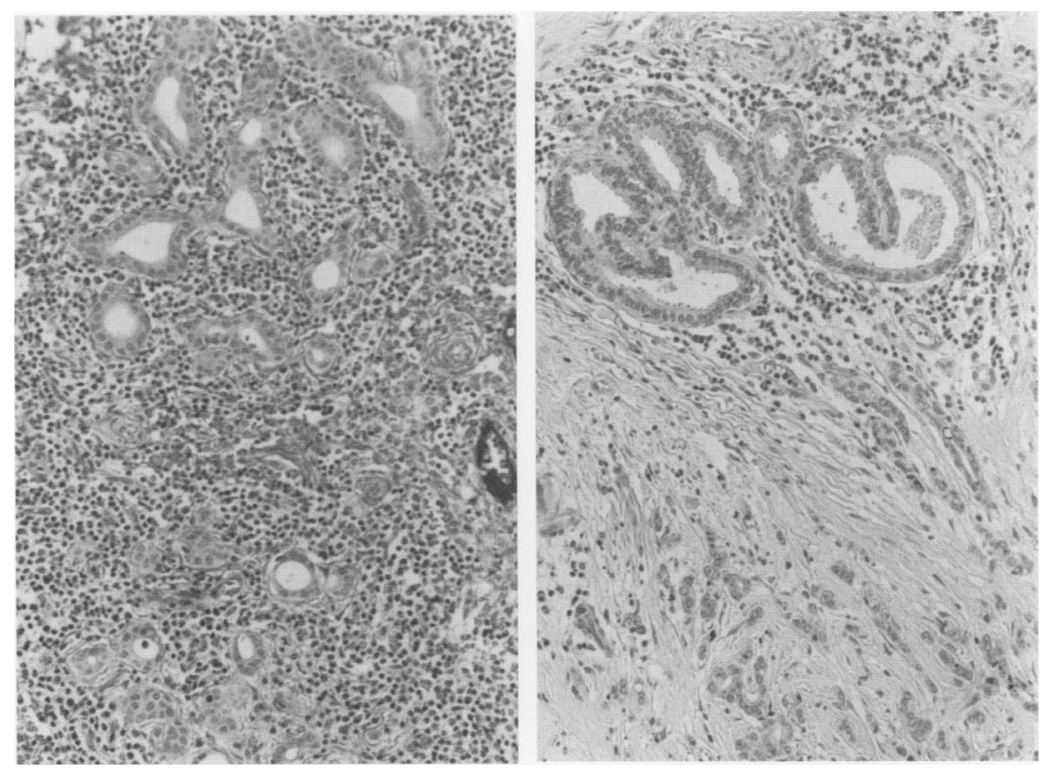

Figure 3 Case 3. (A) Benign epithelial lined tubules within the lymph node; (B) infiltrating carcinoma within ipsilateral breast with adjacent benign glandular elements. tubules and immunohistochemistry showed actin positive cells around each tubule.

CASE 5

A 57 year old woman presented with a $1.2 \mathrm{~cm}$ lump in the right breast which, on biopsy, proved to be an infiltrating lobular carcinoma of classic type, but with focal tubule formation. Two of the 27 lymph nodes removed at subsequent axillary dissection contained solid deposits of metastatic carcinoma. Benign epithelial inclusion cysts were also seen but were restricted to these two nodes.

\section{Discussion}

Large numbers of axillary lymph nodes are regularly routinely examined for metastatic deposits of breast carcinoma. Non-malignant inclusions may be misinterpreted as carcinoma. Small, uniform, round naevus cells arranged in cords and nodules, when present in the capsule of a lymph node, resemble the Indian files of classic infiltrating lobular carcinoma. Rarely, as in case 3, naevus cells are present in the nodal parenchyma as well as the capsule. ${ }^{1}$ Our three cases of naevus cell inclusions were seen in the laboratory within five months, during which time 101 axillary clearances were examined. This high rate of occurrence raised the possibility that such cells might often be missed. To evaluate this we screened 518 lymph nodes from 41 consecutive axillary clearances with $\mathrm{S} 100$ antibody as a marker for naevus cells but found no occult lesions. Ridolfi et al found naevus cell aggregates in $0.33 \%$ of 909 mastectomies. ${ }^{1}$ Interestingly, McCarthy et al reported a higher incidence of $6 \cdot 2 \%$ in lymph node resections, mostly axillary, and most of which were performed for malignant melanoma. $^{2}$

How do naevus cells come to be present in lymph nodes? Johnson and Helwig suggest either an aberration in the migration of naevus cells from the neural crest, or "benign metastases" from a naevus in the skin. ${ }^{3}$ Case 3 is interesting in this context as it showed benign vascular invasion in vessels adjacent to an intradermal naevus as well as in the nodal inclusion. McCarthy et al supported the theory of metastases as they found pigmented naevi in the skin drained by the affected node in 21 of 22 of their cases. ${ }^{2}$

The presence of benign epithelial inclusions in lymph nodes is less common but also well recognised as occurring in patients with benign breast disease or carcinoma.

Epithelial inclusions are often cystic and may be lined by stratified squamous epithelium or show apocrine change. The presence of epithelial hyperplasia and papillomas have both been reported. ${ }^{4}$ Turner and Millis suggested the possibility that malignant change in an epithelial inclusion could account for cases of "metastatic axillary disease" when no primary breast carcinoma can be found. ${ }^{4}$ This possibility is reinforced by a case report by Walker and Fechner, who described a cystic papillary lesion found in an axillary node with 
appearances ranging from benign papilloma to papillary carcinoma. ${ }^{5}$

As with benign naevus cells, the histiogenesis of epithelial inclusions is unknown but, again, both embryological or embolic phenomena have been suggested. Turner and Millis suggested that previous surgical intervention might have precipitated a tumour embolus in their case. ${ }^{4}$ As several of the other reported cases have occurred in women with no previous surgery this seems unlikely.

Naevus cell inclusions can look remarkably like metastatic lobular carcinoma, as seen in two of our cases. Epithelial inclusions arranged in small tubules, as seen in the node from the patient with a tubular mixed carcinoma, can also be misleading. The most worrying aspect of all these benign inclusions is the possibility that they be misdiagnosed as a metastasis. Nowadays we are becoming increasingly reliant on immunohistochemistry in histopathology, both for differential diagnosis and for the detection of small aberrant tissue components such as epithelial cells within lymph nodes. It is important to remember, however, that aberrant tissue may be benign and the judicious use of immunohistochemical markers can help elucidate its true nature.

1 Ridolfi RL, Rosen PP, Thaler H. Nevus cell aggregates associated with lymph nodes: estimated frequency and associated with lymph nodes: estimated freq
clinical significance. Cancer 1977;39:164-71.

2 McCarthy SW, Palmer AA, Bale PM, Hirst E. Nevus cells in lymph nodes. Pathology 1974;6:351-8.

3 Johnson WT, Helwig EB. Benign nevus cells in the capsule of lymph nodes. Cancer 1969;23:747-53.

4 Turner DR, Millis RR. Breast tissue inclusions in axillary lymph nodes. Histopathology 1980;4:631-6.

5 Walker AN, Fechner RE. Papillary carcinoma arising from ectopic breast tissue in an axillary lymph node. Diagnostic Gynecology and Obstetrics 1982;4:141-5.

6 Holdsworth PJ, Hopkinson JM, Leveson SH. Benign axillary epithelial lymph node inclusions-a histological axillary epithelial lymph node inclusior
pitfall. F Clin Pathol 1988;41:226-8. 